\title{
The ROTSE-IIIa Telescope System
}

\author{
D. Smith*, C. Akerlof*, M. C. B. Ashley ${ }^{\dagger}$, D. Casperson**, G. Gisler**, R. Kehoe*, \\ S. Marshall ${ }^{\ddagger}$, K. McGowan**, T. McKay*, M. A. Phillips ${ }^{\dagger}$, E. Rykoff*, W. T. \\ Vestrand $^{* *}$, P. Wozniak ${ }^{* *}$ and J. Wren ${ }^{* *}$ \\ *2477 Randall Laboratory, University of Michigan, 500 E. Univeristy Ave., Ann Arbor, MI, 48109, USA \\ ${ }^{\dagger}$ School of Physics, University of New South Wales, Sydney 2052, Australia \\ ${ }^{* *}$ Los Alamos National Laboratories, Los Alamos, NM, 87545, USA \\ ${ }^{\ddagger}$ Lawrence Livermore National Laboratory, 7000 East Avenue, Livermore, CA, 94550, USA
}

\begin{abstract}
.
We report on the current operating status of the ROTSE-IIIa telescope, currently undergoing testing at Los Alamos National Laboratories in New Mexico. It will be shipped to Siding Spring Observatory, Australia, in first quarter 2002. ROTSE-IIIa has been in automated observing mode since early October, 2001, after completing several weeks of calibration and check-out observations. Calibrated lists of objects in ROTSE-IIIa sky patrol data are produced routinely in an automated pipeline, and we are currently automating analysis procedures to compile these lists, eliminate false detections, and automatically identify transient and variable objects. The manual application of these procedures has already led to the detection of a nova that rose over six magnitudes in two days to a maximum detected brightness of $m_{\mathrm{R}} \sim 13.9$ and then faded two magnitudes in two weeks. We also readily identify variable stars, includings those suspected to be variables from the Sloan Digital Sky Survey. We report on our system to allow public monitoring of the telescope operational status in real time over the WWW.
\end{abstract}

\section{TELESCOPE STATUS}

ROTSE-IIIa is a $0.45-\mathrm{m}$ robotic reflecting telescope which is managed by a fully-automated system of interacting daemons within a Linux environment. The telescope has an f-ratio of 1.9, yielding a field of view (FOV) of $1.85 \times 1.85$ degrees. The control system is connected via a TCP/IP socket to the Gamma-Ray Burst Coordinate Network (GCN), and a flexible scheduler daemon plans observation sequences including sky patrols, targeted monitoring programs, and fast $(<10 \mathrm{~s})$ responses to GRB alerts. Upon receipt of a GRB alert over the GCN, we begin a program of 105 -s, 1020 -s, and 80 60 -s exposures (with a read-out time of $7 \mathrm{~s}$ between images). We also automatically schedule blocks of 30 60-s follow-up exposures. These blocks are spaced at everincreasing intervals. The system began its testing run on October 11, 2001, at the ROTSE-I site at Los Alamos National Laboratory. ROTSE-IIIa will be shipped to Siding Springs Observatory, Australia, upon completion of the testing phase.

ROTSE-IIIa currently uses unfiltered CCDs, although we have included a slot in the mechanical design that will allow for the insertion of a filter. We currently calibrate ROTSE magnitudes against the USNO R-band magni- tudes, and we include a constant offset of 0.3 magnitudes to convert these numbers to a V-band magnitude. This necessarily introduces unknown systematic errors for objects with atypical spectra. As shown in Figure 1, ROTSE-IIIa can reach 17 th magnitude in a 5-s exposure, 17.5 in $20-\mathrm{s}$, and 18.5 in a 60 -s exposure. Longer exposures are not practical due to saturation of the sky. Multiple images can be co-added to reach $\sim 19$ th magnitude.

Figure 2 shows the dispersion of calibrated stellar magnitudes over time (corrected to $\mathrm{V}$ band). Plotted are the standard deviations vs. the mean magnitude of $\sim 3000$ objects detected in at least 10 of 20 observations of a single field. The intensity of bright stars varies by less than $1 \%$, while the RMS deviation rises to $\sim 30 \%$ for the dimmest stars ROTSE-IIIa can detect.

To measure the astrometric accuracy of our analysis, we compare the relative positions of objects found in both the USNO A2.0 catalog as well as in the abovementioned 20 ROTSE-IIIa observations. The transformation of the CCD coordinates to celestial coordinates is achieved through a third-order polynomial warp. Most bright stars $\left(m_{\mathrm{V}}<14\right)$ can be localized to better than 0.3 arcsec, or one-tenth of a pixel, and the bulk of the faintest objects stay within two-thirds of a pixel.

CP662, Gamma-Ray Burst and Afterglow Astronomy 2001: A Workshop Celebrating the First Year of the HETE Mission,

edited by G. R. Ricker and R. K. Vanderspek

(C) 2003 American Institute of Physics 0-7354-0122-5/03/\$20.00 


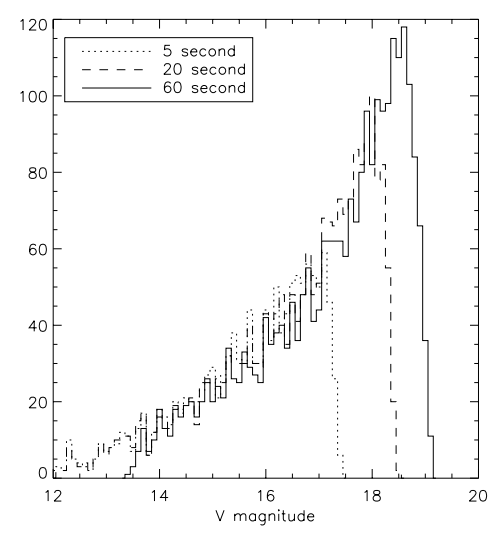

FIGURE 1. Number of objects per magnitude bin for a random ROTSE-IIIa field at three exposure times. Magnitudes were derived by calibrating to the USNO R-band and then applying a constant correction factor of 0.3 mag to convert to an approximate V-band.

\section{DATA ANALYSIS PIPELINE}

We are implementing software to automatically analyze all images recorded with the ROTSE-III telescopes. The full analysis pipeline for each telescope will be installed on a computer at each site, but during testing we are copying most of the data to the University of Michigan for easier control. All images are automatically dark- and flat-field corrected as soon as they are recorded. Immediately after each corrected image file is written, it is processed into an object list with the SExtractor package [1], using an aperture 5 pixels in diameter, to identify all source candidates within the FOV. The list of these candidate objects is compared against the USNO A2.0 catalog using a triangle-matching routine to compile a calibrated list of R-band magnitudes and celestial locations for these sources. Calibrated object lists also allow diagnostic parameters to be measured, such as the astrometric accuracy and the focus quality. These steps have been implemented on an on-site computer and the system produces a calibrated object list for each image within $45 \mathrm{~s}$ from the closing of the shutter.

Once more than one calibrated list for a given field is available, these lists are compiled into a "match structure"; a data structure that enables us to filter out objects that do not appear at the same location in sequential observations. The telescope aspect is shifted through a small, random vector between observations. This enables the elimination of hot CCD pixels and cosmic ray events. We then apply a relative photometry algorithm to stabilize the magnitude estimates and calibrate the systematic errors. This reduces the scatter in light curves for stable bright objects to $<1 \%$ (Fig. 2) and allows us to reliably identify variable sources. We can also flag known

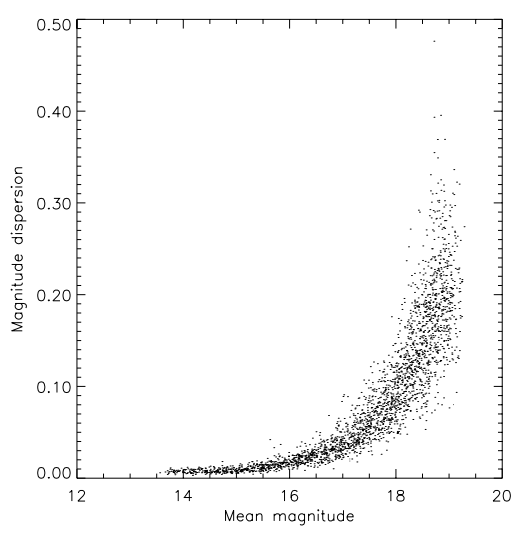

FIGURE 2. Photometric scatter in 20 60-s observations of 3,000 objects over a two-week interval as a function of V-band magnitude. The images have been corrected using a relative photometry algorithm. Root mean square deviations vary from $\sim 1 \%$ at 14 th magnitude to $\sim 10-30 \%$ at the magnitude limit.

distractions such as asteroids and "masked" stars (stars that appear in ROTSE images but are too close to bright stars for sensitive cataloged surveys to resolve). Once this procedure is automated, we anticipate that the system will be able to report an arcsecond position for a bright $\left(m_{\mathrm{R}}>17\right)$, variable object within five minutes of the receipt of a burst trigger via a GCN alert.

\section{DISCOVERIES}

Calibrated match structures can easily be searched for previously unknown objects. For our first testing of the procedures involved, we constructed these structures for 20 epochs over two weeks for $\sim 500$ sq. deg. of sky patrols along the Equatorial strips of the Sloan Digital Sky Survey Early Data Release. Variable stars can be identified through application of the techniques developed for ROTSE-I [2]. In Figure 3, we show the folded light curve for a typical RR Lyra star in a ROTSE-IIIa field. The best-fit period of $0.64 \mathrm{~d}$ was found using a cubic spline method [3]. This method provides a best-fit period and error estimate for a variable star light curve, as well as a spline-interpolated approximation for the source light curve.

Non-periodic variables can also be identified. Figure 4 shows the light curve for a new transient that was not detected on Oct 11.424, 2001 (with a limiting magnitude of 18.2), but was easily visible at a magnitude of 14.0 on Oct 13.291, $45 \mathrm{~h}$ later. Clouds prevented observations on the night of Oct 12. The transient then faded two magnitudes over the subsequent two weeks until proximity to the waxing moon interfered with further monitoring. 


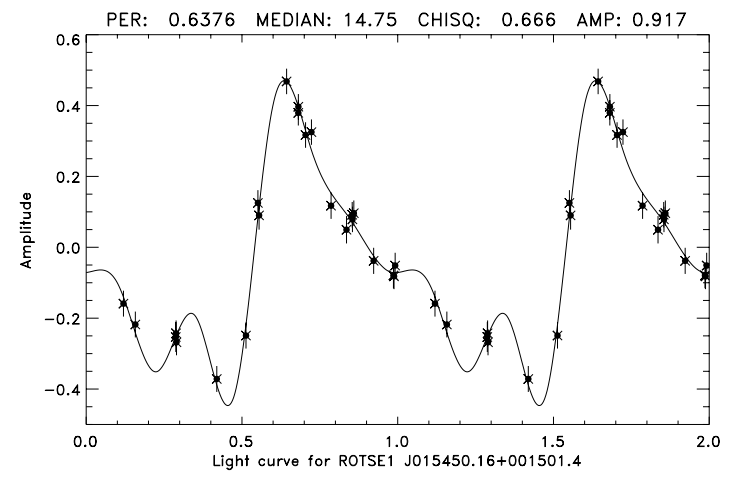

FIGURE 3. Folded light curve in calibrated R-band for an identified variable star in one of the ROTSE-IIIa sky patrol fields. An estimate of a systematic error of $4 \%$ has been added in quadrature to the statistical errors. The mean magnitude of 14.75 has been subtracted.

The best-fit power law index for the fading light curve is $\sim 0.9$, but the shape of the curve is clearly not consistent with a single power law. With a mean magnitude of 15 and an RMS deviation around that mean of 0.66, this source stood out starkly against the typical behavior of other stars (Fig. 2). The ease with which this source was detected is a positive indication of the potential for using ROTSE-IIIa to identify and track new transients.

\section{REALTIME WWW MONITORING}

The telescope operating system writes all values of its status variables to a file once every minute. These variables include the current pointing direction, the name of the most recent image, whether the enclosure roof is open or closed, the current velocity of the mount along its two axes, and whether or not any alarms are active. This file is copied back to the University of Michigan by a cron job and parsed into an HTML file that can be viewed in real time over the WWW at http://wWw. rotse. net. Also included in this display are a thumbnail of the most recent image and graphs of the diagnostic parameters derived from the calibrated object lists. This interface allows interested parties to monitor burst response and telescope status in near-real-time.

\section{CONCLUSIONS}

ROTSE-IIIa is working well in its first testing phase at Los Alamos. Operation and image processing has been automated, and image analysis is being automated at the time of this writing. When full automation is implemented, we will be able to report an arcsecond

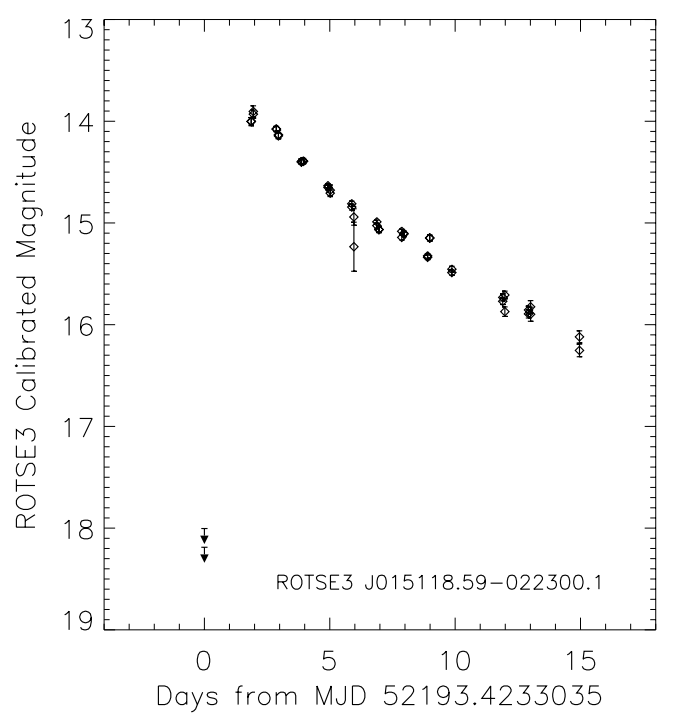

FIGURE 4. Light curve in the calibrated R-band for a transient nova discovered in the first two weeks of automated sky patrols by ROTSE-IIIa. Arrows indicate the limiting magnitudes of two images taken $1.8 \mathrm{~d}$ before the first detection at $m_{\mathrm{R}}=14.00 \pm 0.04$ on Oct $13.291,2001$. The source was easily detectable for the following two weeks, until proximity to the waxing moon interfered with continued monitoring.

position for a bright $\left(m_{\mathrm{R}}>17\right)$, rapidly variable $(\Delta m>$ $10 \%$ within the first minute) object within five minutes of receipt of a burst trigger. Limiting magnitudes under good conditions in 60-s exposures can approach 19th magnitude, and we anticipate gaining further sensitivity at the (darker) permanent site in Australia.

\section{ACKNOWLEDGMENTS}

D. Smith is supported by NSF fellowship 00-136. Work performed at LANL is supported by NASA SR\&T through Department of Energy (DOE) contract W-7405ENG-36 and through internal LDRD funding. Work performed at the University of Michigan is supported by NASA under SR\&T grant NAG5-5101, the NSF under grants AST 97-03282 and AST 99-70818, the Research Corporation, the University of Michigan, and the Planetary Society. Work performed at LLNL is supported by NASA SR\&T through DOE contract W-7405-ENG-48.

\section{REFERENCES}

1. Bertin, E., and Arnouts, S., $A \& A S$, 117, 393-404 (1996).

2. Akerlof, C., et al., AJ, 119, 1901-1913 (2000).

3. Akerlof, C., et al., ApJ, 436, 787-794 (1994). 